\title{
Measurements, Analysis, and Modeling of BitTorrent-like Systems
}

\author{
Lei Guo ${ }^{1}$, Songqing $\mathrm{Chen}^{2}$, Zhen Xiao ${ }^{3}$, Enhua Tan ${ }^{1}$, Xiaoning Ding ${ }^{1}$, and Xiaodong Zhang ${ }^{1}$ \\ ${ }^{1}$ Department of Computer Science \\ College of William and Mary \\ ${ }^{2}$ Department of Computer Science \\ George Mason University \\ Williamsburg, VA 23187, USA \\ Fairfax, VA 22030, USA \\ sqchen@cs.gmu.edu \\ ${ }^{3}$ AT\&T Labs-Research \\ 180 Park Ave. \\ Florham Park, NJ 07932, USA \\ xiao@ research.att.com
}

\begin{abstract}
Existing studies on BitTorrent systems are single-torrent based, while more than $85 \%$ of all peers participate in multiple torrents according to our trace analysis. In addition, these studies are not sufficiently insightful and accurate even for single-torrent models, due to some unrealistic assumptions. Our analysis of representative BitTorrent traffic provides several new findings regarding the limitations of BitTorrent systems: (1) Due to the exponentially decreasing peer arrival rate in reality, service availability in such systems becomes poor quickly, after which it is difficult for the file to be located and downloaded. (2) Client performance in the BitTorrentlike systems is unstable, and fluctuates widely with the peer population. (3) Existing systems could provide unfair services to peers, where peers with high downloading speed tend to download more and upload less. In this paper, we study these limitations on torrent evolution in realistic environments. Motivated by the analysis and modeling results, we further build a graph based multi-torrent model to study inter-torrent collaboration. Our model quantitatively provides strong motivation for inter-torrent collaboration instead of directly stimulating seeds to stay longer. We also discuss a system design to show the feasibility of multi-torrent collaboration.
\end{abstract}

\section{Introduction}

BitTorrent [8] is a new generation of Peer-to-Peer (P2P) system that has become very popular recently. According to a recent $\mathrm{CNN}$ report, BitTorrent traffic represents $53 \%$ of all P2P traffic on the Internet in June 2004 [16]. Unlike traditional P2P systems such as Gnutella [1], KaZaa [2], and eDonkey/eMule/Overnet [3], in which peers sharing different files are organized together and exchange their desired files with each other, BitTorrent organizes peers sharing the same file into a P2P network and focuses on fast and efficient replication to dis- tribute the file. In BitTorrent, a file is divided into small chunks, and a peer can download multiple chunks of the file in parallel. Peers with different file chunks are stimulated to exchange with each other through a "titfor-tat" incentive mechanism, which enables peers with high uploading bandwidth to have corresponding high downloading bandwidth. In this way, BitTorrent prevents free riding effectively, which is very common in early P2P systems [5]. P2P systems for exchanging different files such as KaZaa and eMule use participation levels or credit/reputation systems to track the contribution of each peer, and encourage peers to contribute by giving higher service priority to those peers with more contribution. However, such systems are either too complex and unrealistic or very easy to be circumvented $[4,6]$. Compared to these systems, the direct "tit-for-tat" mechanism of BitTorrent is very simple and effective. In practice, BitTorrent-like systems scale fairly well during flash crowd period and are now widely used for various purposes, such as for distributing large software packages $[7,14]$.

Research has been conducted to study the effectiveness of BitTorrent-like systems [7, 14, 17, 18, 23]. The most recent work shows the stability of BitTorrent-like systems through a fluid model, and verifies the effectiveness of the current incentive mechanism [18]. However, this fluid model assumes a Poisson arrival model for the requests, which has been shown to be unrealistic during a long period (eight months) of trace study [17]. Consequently, the model can only characterize the performance of the BitTorrent system under stable conditions. In reality, as shown by our trace analysis, the stable period is very short. In addition, all existing studies on BitTorrent-like systems focus on the behaviors of single-torrent systems only, while our traces show that most peers $(>85 \%)$ participate in multiple torrents.

In this work, we present an extensive study of BitTorrent-like P2P systems through measurements, trace analysis, and modeling. We first study the evolu- 
tion of BitTorrent systems based on realistic assumptions analyzed from traces. We find that although the existing system is effective to address the "flash crowd" problem upon the debut of a new file, it has the following limitations:

- Due to the exponentially decreasing peer arrival rate and the lack of seeds (peers with a full copy of the file), service availability in the BitTorrent system becomes poor quickly, after which it is difficult for the file to be located and downloaded.

- Client performance in the BitTorrent system is unstable and fluctuates substantially with peer population variations.

- Existing systems can provide unfair services to peers. Studying the peer contribution ratio (uploaded bytes over downloaded bytes), we find that the peer contribution ratio decreases with its downloading speed.

Motivated by the results for the single-torrent system, we further study the multi-torrent system through trace analysis and modeling. Although it was generally understood that collaboration among multiple torrents might overcome some of the limitations of the single-torrent system, to our best knowledge, our work is the first to quantitatively and comprehensively analyze the multitorrent system. In detail, we (1) characterize the peer request pattern in multiple torrents; (2) study the service potentials a torrent can provide to and get from other torrents; (3) demonstrate the benefit of inter-torrent collaboration. In addition, we discuss a new architecture to facilitate inter-torrent collaboration and show the feasibility and compatibility to the current BitTorrent systems.

Our contributions in this work are:

- We find three limitations of existing BitTorrent-like systems through torrent evolution study based on correct peer arrival pattern.

- Motivated by the modeling and analysis results, we build a graph-based multi-torrent model to quantify the inter-torrent collaboration benefit. The result shows that inter-torrent collaboration is much more effective than directly stimulating seeds to stay longer, addressing the well-known problem of lacking incentives to seeds.

- Guided by the modeling result, we propose and discuss a new architecture for inter-torrent collaboration.

The remainder of the paper is organized as follows. Section 2 presents related work. We demonstrate the limitations of existing BitTorrent-like systems through measurements, trace analysis, and modeling in Section 3, and present our multi-torrent model in Section 4. Section 5 proposes and discusses an architecture for intertorrent collaboration. We make concluding remarks in Section 6.

\section{Other Related Work}

The amount of $\mathrm{P} 2 \mathrm{P}$ traffic and the population of $\mathrm{P} 2 \mathrm{P}$ users on the Internet keeps increasing. A lot of studies have been performed on the measurements, modeling, and algorithms of different $\mathrm{P} 2 \mathrm{P}$ systems.

Measurement studies $[19,20]$ characterize the P2P traffic over the Internet, including Napster, Gnutella, and KaZaa systems. Study [12] analyzes the popularity of $\mathrm{P} 2 \mathrm{P}$ content over the Internet and characterizes the "download at most once" property of P2P clients. Extensive measurements and traffic analysis on BitTorrent systems have also been conducted recently. Study [14] analyzes a five-month workload of a single BitTorrent system for software distribution that involved thousands of peers, and assesses the performance of BitTorrent at the flash crowd period. In [7], authors analyze the BitTorrent traffic of thousands of torrents over a two-month period, with respect to file characteristics and client access characteristics. Work [17] presents the current infrastructure of BitTorrent file sharing systems, including the Web servers/mirrors for directory service, meta-data distribution, and $\mathrm{P} 2 \mathrm{P}$ content sharing. The authors also find that the arrival, abort, and departure processes of downloaders do not follow a Poisson distribution in the eight-month trace they collected, which was assumed in the previous modeling study [18].

A queuing model for P2P file sharing systems is proposed in [11]. Study [23] analyzes the service capacity of BitTorrent-like systems, and finds that multi-part downloading helps P2P systems to improve performance during flash crowd period. Study [18] further characterizes the overall performance of BitTorrent-like systems using a simple fluid model, and analyzes the effectiveness of BitTorrent incentive mechanism using game theory. Study [15] introduces a probabilistic model of coupon replication systems, and analyzes the performance under an environment where neither altruistic user behaviors nor load balancing strategies (such as rarest first in BitTorrent) are supported.

Study [22] proposes an interest-based content location approach for P2P systems. By self-organizing into small groups, peers with the same interest can collaborate more efficiently, which is similar to the BitTorrent networks, where all peers share the same file. In [21], a P2P protocol is proposed for bulk data transfer, which aims to improve client performance and to reduce server load, by using enhanced algorithms over BitTorrent systems. 
Different from all studies above, our modeling and trace analysis provide an understanding of torrent evolution in the BitTorrent systems and the relation among multiple torrents over the Internet. Furthermore, our results reveal three limitations in current BitTorrent systems, and propose an innovative architecture to facilitate inter-torrent collaboration, which represents the first step towards making the current BitTorrent-like system a reliable and efficient content delivery vehicle.

\section{Modeling and Characterization of BitTorrent-like Systems}

In a BitTorrent system, the content provider creates a meta file (with the . torrent suffix name) for the torrent file it wants to share, and publishes the meta file on a Web site. Then the content provider starts a BitTorrent client with a full copy of the torrent file as the original seed. For each torrent file, there is a tracker site, whose URL is encoded in the meta file, to help peers find each other to exchange the file chunks. A user starts a BitTorrent client as a downloader at the beginning to download file chunks from other peers or seeds in parallel. A peer that has downloaded the file completely also becomes a seed that could in turn provide downloading service to other peers. All peers in the system, including both downloaders and seeds, self-organize into a P2P network, known as a torrent. The initial seed can leave the torrent when there are other seeds available, and content availability and system performance in the future depend on the arrival and departure of downloaders and other seeds.

Previous research has studied BitTorrent-like systems through trace analysis and modeling, and verified its effectiveness during flash crowds, which normally happen soon upon the debut of a new file [18]. However, no existing work has characterized overall client performance in the lifetime of a torrent when the file popularity changes. This is particularly important for BitTorrentlike systems where service availability relies purely on the voluntary participation of peers. This is in contrast to a client-server model where a permanent site (i.e., a server) can provide persistent service.

In this section, we study torrent evolution, downloading service availability, client performance fluctuation, and service fairness in BitTorrent-like systems based on torrent popularity characterization. We propose an evolution model for BitTorrent-like systems and analyze the torrent lifespan, ratio of failed peers, and the service policy of seeds, based on both the modeling and trace analysis.

\subsection{Torrent Popularity Characterization}

In this study, we analyze and model BitTorrent traffic based on two kinds of traces. The first one contains the statistics collected from two popular dedicated tracker sites (although each torrent can have its own tracker site, there are many dedicated tracker sites on the Internet providing persistent service, each of which may host thousands of torrents), sampled every half an hour for 48 days from 2003-10-23 to 2003-12-10. This trace was collected by University of Massachusetts, Amherst [7] (abbreviated as the tracker trace or trace in the remainder of this paper). We identify different peers and match multiple sessions of the same downloading with the similar methods used in study [14]. The firewalled peers, although they cannot accept incoming connections and thus are not listed in the tracker query responses to allow other peers to connect to, are still included in the tracker statistics. We extract the peer request time, downloading/uploading bytes, the downloading/uploading bandwidth of all peers for each torrent, and the information for each torrent such as torrent birth time and file size. Due to space limitations, we only present the analysis results of the larger tracker trace, which includes more than 1,500 torrents (about 550 torrents are fully traced during their lifecycles). The smaller trace has similar results.

In order to better understand BitTorrent traffic over the Internet, we also collected the BitTorrent meta file downloading trace from a large commercial server farm hosted by a major ISP and a large group of home users connected to the Internet via a well-known cable company, using the Gigascope appliance [10], from 200409-28 to 2004-10-07. The server farm trace includes about 50 tracker sites hosting hundreds of torrents, and the cable network trace includes about 3,000 BitTorrent users (by IP addresses) requesting thousands of torrents on the Internet. Both traces include the first IP packets of all HTTP downloading of the . torrent files, with the timestamp when the packet is captured (the downloading time of the .torrent file). This timestamp represents the peer arrival time to the torrent. We also extract the timestamp encoded in each . torrent file, which is the creation time of the meta file and represents the torrent birth time.

Figure 1(a) shows the complementary CDF (CCDF) distribution of the time after torrent birth for the requests to all fully-traced torrents in the tracker trace. For peers downloading the file in multiple sessions, only the first requests are considered. The $y$-axis at time $t$ denotes the total number of requests for all torrents in the trace minus the cumulative number of requests for all torrents after time $t$ since they are born. Figures 1(b) and 1(c) show the CCDF distribution of the time when a torrent file was downloaded after torrent birth in the server farm 


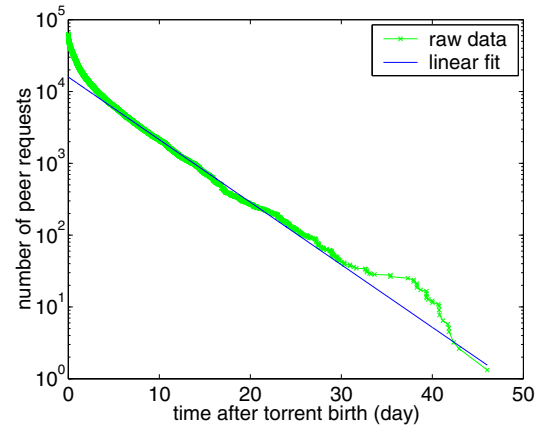

(a) Tracker trace

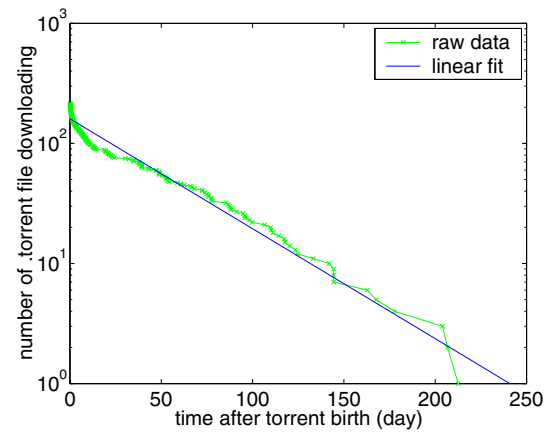

(b) Server farm trace

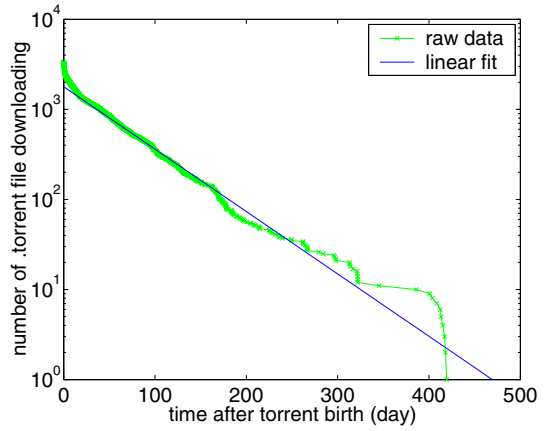

(c) Cable network trace

Figure 1: The complementary CDF distribution of peer arrival time (time of a peer's first request to a torrent or time when a meta file was downloaded) after torrent birth for three BitTorrent traces ( $y$-axis is in log scale)

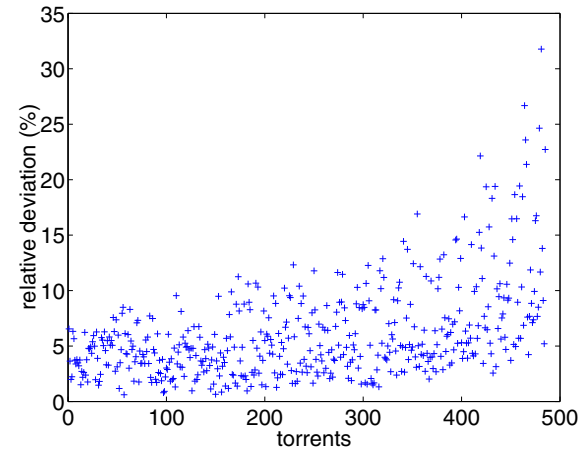

Figure 2: Fitting deviations of fully-traced torrents in the tracker trace

and in the cable network, respectively. Note that $y$-axis is in log scale in the three figures.

All three curves can be fitted with straight lines. This consistent trend strongly suggests that after a torrent is born, the number of peer arrivals to the torrent decreases exponentially with time in general. To validate that this conjecture holds for individual torrents as well, we use the least square method to fit the logarithm of the complementary of the number of peer arrivals for each torrent in the tracker trace. We define the relative deviation of the fitting for the number of requests at a time instant as $\frac{\left|\log N_{0}-\log N\right|}{\log N_{0}} \times 100 \%$, where $N_{0}$ is the actual complementary value of the number of requests and $N$ is the fitting result. Figure 2 shows the distribution of average fitting deviation for each fully-traced torrent that has at least 20 peers during its lifetime. In this figure, each point in the $x$-axis denotes a torrent, sorted in nonascending order of torrent population during the entire lifetime, and the corresponding value in $y$-axis denotes the average of relative fitting deviation of this torrent. We can see the fitting is more accurate for torrents with larger population, and the overall average relative deviation is only about $6 \%$. We do not fit the curve for individual torrents in the server farm and cable network trace, because the data collection duration is short so that they do not cover the whole lifespans of torrents. In the remainder part of this paper, we only use the tracker trace for modeling and analysis.

We define the torrent popularity at a time instant as the peer arrival rate of the torrent at that time, which is the derivative of the peer arrival time distribution of that torrent. Since the derivative of an exponential function is also an exponential function, we assume that the peer arrival rate of a torrent follows an exponential decreasing rule with time $t$

$$
\lambda(t)=\lambda_{0} e^{-\frac{t}{\tau}}
$$

where $\lambda_{0}$ is the initial arrival rate when the torrent starts, and $\tau$ is the attenuation parameter of the torrent evolution. In Section 3.3, we will use a fluid model to evaluate our assumption again.

\subsection{Evolution and Service Availability of BitTorrent}

We define the torrent lifespan as the duration from the birth of the torrent to the time after which there is no complete copy of the file in the system, and the new arriving peers cannot complete downloading. To simplify the modeling, we assume that the initial seed exits the system as soon as a downloader has downloaded the file completely. In practice, the initial seed may stay online in the system for a longer time, and some seeds may return to the system to serve the content.

The inter-arrival time between two successive arriving peers $\delta t$ can be approximated as $\frac{1}{\lambda}$. Denote the rate at which seeds leave the system as $\gamma$, then the average service time of a seed can be approximated as $\frac{1}{\gamma}$. As shown in Figure 3, peer $n$ and peer $n+1$ are the $n$-th and $(n+1)$ th arriving peers in the torrent, at the time $t_{n}$ and $t_{n+1}$, 


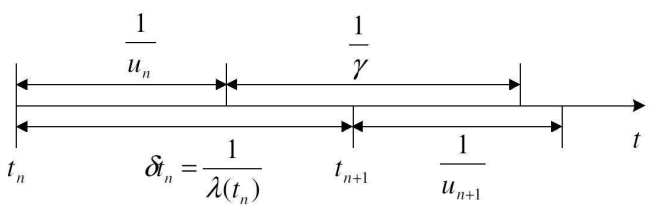

Figure 3: The death of a torrent due to large inter-arrival time of peers

respectively. The inter-arrival time between peer $n$ and peer $n+1$ can be estimated as $\delta t_{n}=t_{n+1}-t_{n} \approx \frac{1}{\lambda\left(t_{n}\right)}$. Peer $n$ downloads the file with speed $u_{n}$ and then stays in the torrent for a time duration $\frac{1}{\gamma}$. Peer $n+1$ downloads at speed $u_{n+1}$. According to the exponential decrease of peer arrival rate, the inter-arrival time of peers will grow exponentially, and finally there will be only one seed at a time. When the peer arrival rate $\lambda(t)$ is small enough ( $n$ is large), peer $n+1$ can only be served by peer $n$, and we have $u_{n+1} \leq u_{n}$. Thus, when $\delta t_{n} \approx \frac{1}{\lambda\left(t_{n}\right)}>\frac{1}{\gamma}$, peer $n+1$ cannot complete downloading before peer $n$ leaves, and the torrent is dead. Using Equation 3.1, we get the torrent lifespan

$$
T_{l i f e}=\tau \log \left(\frac{\lambda_{0}}{\gamma}\right) .
$$

Equation 3.2 shows the expectation of the real torrent lifespan. To verify Equation 3.2, we compute the initial peer arrival rate $\lambda_{0}$ and the torrent attenuation parameter $\tau$ for fully traced torrents in the tracker trace. From Equation 3.1, we have

$$
\log \delta t=-\log \lambda_{0}+\frac{t}{\tau} .
$$

Both $\delta t$ and $t$ for each peer arrival can be extracted from the trace and we get $\log \lambda_{0}$ and $\frac{1}{\tau}$ using linear regression. We also compute the seed leaving rate $\gamma$ as the the reciprocal of the average seed service time, which is extracted from the trace, too. Figure 4 shows the comparison of torrent lifespan computed from the tracker trace (indicated by trace) and that from the Equation 3.2 (indicated by model). In this figure, each point in $x$-axis denotes a torrent, while each point in $y$-axis denotes the measurement result or the modeling result of torrent lifespan. The torrents in the $x$-axis are sorted in non-ascending order of the modeling results of torrent lifespans. As shown in the figure, our model fits the real torrent lifespan very well. The average lifespan of torrents is about 8.89 days based on the trace analysis and 8.34 days based on our model. The lifespans of most torrents are between 30 - 300 hours, and there are only a small number of torrents with extremely short or extremely long lifespans.

The total population of a torrent (in the number of

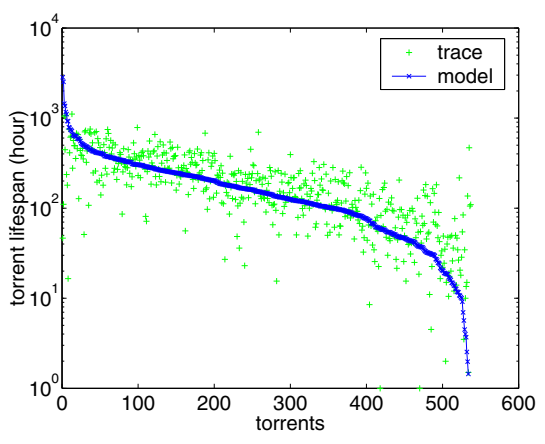

Figure 4: The comparison of torrent lifespan: modeling and trace analysis ( $y$-axis is in log scale)

peers) is

$$
N_{\text {all }}=\int_{0}^{\infty} \lambda_{0} e^{-\frac{t}{\tau}} d t=\lambda_{0} \tau .
$$

Among them, some peers may not be able to complete downloading due to lack of seeds, which we call failed peers, denoted as follows:

$$
N_{\text {fail }}=\int_{T_{\text {life }}}^{\infty} \lambda_{0} e^{-\frac{t}{\tau}} d t=\gamma \tau .
$$

Thus, the downloading failure ratio of the torrent is

$$
R_{\text {fail }}=\frac{N_{\text {fail }}}{N_{\text {all }}}=\frac{\gamma \tau}{\lambda_{0} \tau}=\frac{\gamma}{\lambda_{0}} .
$$

Figure 5(a) shows the comparison of the torrent population computed from the tracker trace with that from our model for each individual fully-traced torrent. In this figure, each point in $x$-axis denotes a torrent, while each point in $y$-axis denotes the measurement result or the modeling result of the total population of the torrent during its entire lifespan. The torrents in the $x$-axis are sorted in non-ascending order of the modeling results of torrent populations. As evidenced by the figure, the modeling result and trace analysis are consistent. In addition, we can see that the distribution of the torrent population is heavily skewed: although there are several large torrents, most torrents are very small, and the average population of torrents is only about 102 peers.

Figure 5(b) shows the downloading failure ratio based on trace analysis and on our model (plotted in the similar manner as that of Figure 5(a)). The real failure ratio of torrents is slightly lower than what our model predicts, because there are some altruistic peers that serve the torrent voluntarily. That also explains why the torrent lifespan in the trace analysis (8.89 days) is slightly higher than that in our model (8.34 days). Furthermore, there are some torrents that have no failed peers in the trace because the seeds leave after the downloaders finish, but 


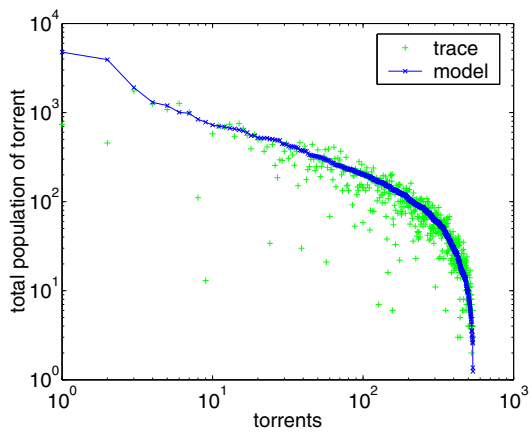

(a) The comparison of torrent population: modeling and trace analysis (in log-log scale)

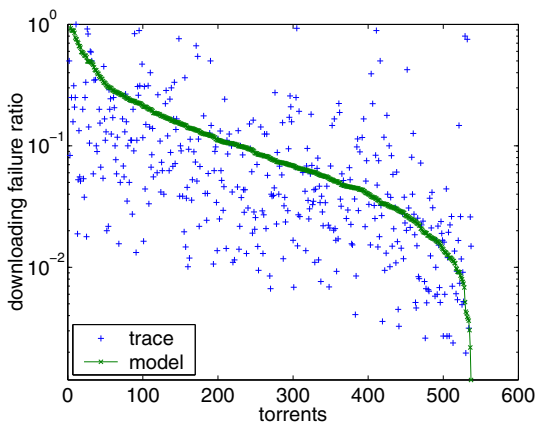

(b) The comparison of downloading failure ratio: modeling and trace analysis ( $y$-axis is in $\log$ scale)

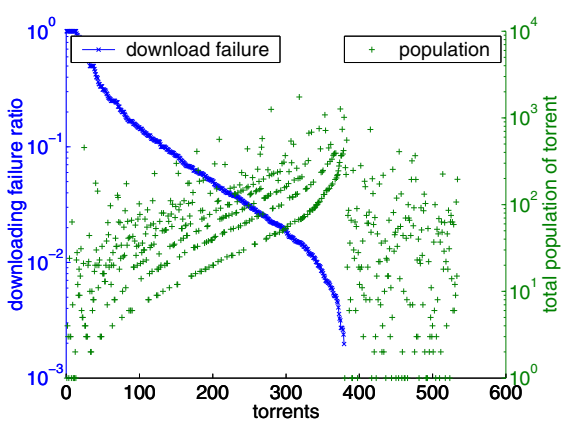

(c) The relation between torrent population and downloading failure ratio ( $y$-axis is in log scale)

Figure 5: Torrent population and downloading failure ratio for all fully-traced torrents

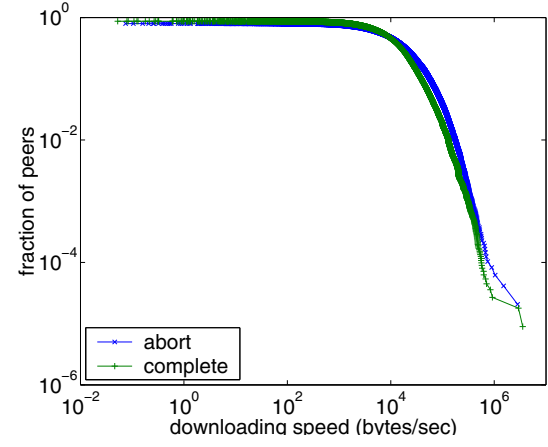

(a) The downloading speed distribution (complementary CDF, in log-log scale)

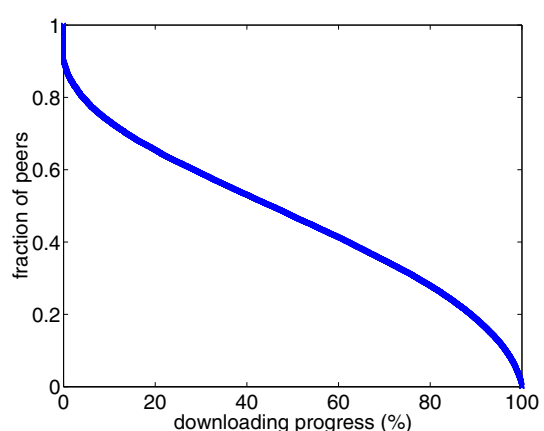

(b) The downloading progress distribution (complementary CDF)

Figure 6: The peers abort downloading voluntarily

cannot be shown in the log scale plot. However, the average downloading failure ratio based on the trace analysis is still about $10 \%$, which is non-trivial for a content distribution system.

Equation 3.5 implies that the number of failed peers in a torrent is independent of the initial peer arrival rate. Instead, the number of failed peers depends on the speed of torrent evolution (the attenuation exponent of peer arrival rate) and the seed departure rate. Figure 5(c) shows downloading failure ratios of torrents and their corresponding populations (plotted in the similar manner as that of Figure 5(a) and 5(b)). As reflected in the figure and indicated by Equation 3.5, the larger the torrent population, the lower the downloading failure ratio. It is interesting to note that the population of torrents, sorted in non-ascending order of their corresponding downloading failure ratios, forms several clear curves, each of which represents those torrents with similar evolution patterns (the popularity attenuation parameter $\tau$ ). On the right side of the figure, the failure ratio of the torrents is 0 due to the existence of some altruistic seeds, which always stay until the last downloader completes.
In the above analysis, we assume that peers always complete their downloading unless they cannot. We do not consider peers that abort downloading voluntarily when seeds are still available in the torrent. A peer may abort downloading due to (1) loss of interest to the torrent file; (2) slow downloading speed or small downloading progress. Figure 6(a) shows the distribution of the average downloading speed of peers that voluntarily abort and peers that download the file completely. Figure 6(b) shows the distribution of downloading progress (the percentage of the entire file that has been downloaded) when peers abort downloading voluntarily. The figures indicate that the probability for a peer to abort downloading voluntarily is almost independent of its downloading speed and the current downloading progress. Hence, the voluntary aborting of some downloaders does not affect our analysis above. 

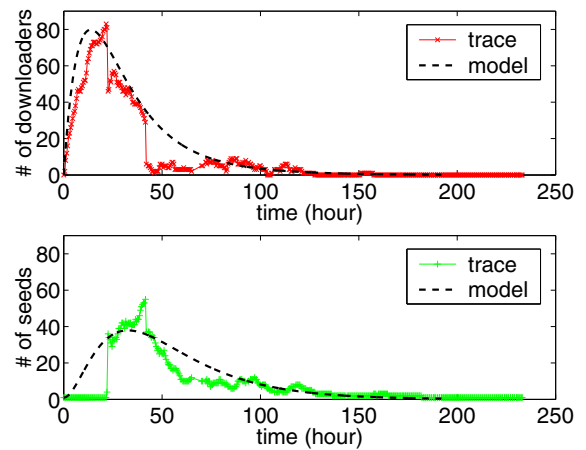

(a) Torrent evolution

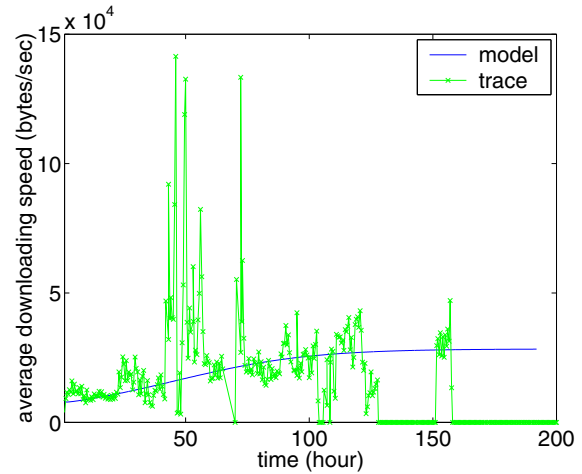

(b) Downloading speed

Figure 7: Torrent evolution under the fluid model

\begin{tabular}{|c|l|}
\hline$x(t)$ & number of downloaders in the system at time $t$ \\
\hline$y(t)$ & number of seeds in the system at time $t$ \\
\hline$\lambda_{0}$ & the initial value of peer arrival rate \\
\hline$\tau$ & the attenuation parameter of peer arrival rate \\
\hline$\mu$ & the uploading bandwidth \\
\hline$c$ & the downloading bandwidth $(c \gg \mu)$ \\
\hline$\gamma$ & the rate at which seeds leave the system \\
\hline$\eta$ & $\begin{array}{l}\text { the file sharing efficiency, meaning the probability } \\
\text { that a peer can exchange chunks with other peers }\end{array}$ \\
\hline
\end{tabular}

Table 1: Notations and assumptions for the fluid model

\subsection{Client Performance Variations in Bit- Torrent}

Study [18] proposed a fluid model for BitTorrent-like systems with constant peer arrival rate. We follow the idea of the fluid model, but using the evolution of peer arrival rate described in Equation 3.1. The basic ODE (ordinary differential equation) set for the fluid model is

$$
\left\{\begin{array}{l}
\frac{d x(t)}{d t}=\lambda_{0} e^{-\frac{t}{\tau}}-\mu(\eta x(t)+y(t)), \\
\frac{d y(t)}{d t}=\mu(\eta x(t)+y(t))-\gamma y(t) \\
x(0)=0, y(0)=1
\end{array}\right.
$$

where the meanings of the parameters in our fluid model are listed in Table 1. These notations are adopted from work [18, 23].

When the ODE set has two different real eigenvalues $\psi_{1} \neq \psi_{2}$, the resolution can be expressed as:

$$
\left\{\begin{array}{l}
x(t)=a e^{\psi_{1} t}+b e^{\psi_{2} t}+d_{1} e^{-\frac{t}{\tau}}, \\
y(t)=c_{1} a e^{\psi_{1} t}+c_{2} b e^{\psi_{2} t}+d_{2} e^{-\frac{t}{\tau}},
\end{array}\right.
$$

where $d_{1}, d_{2}, c_{1}, c_{2}, a, b$ are constant. The value of these constants and the detailed resolution of the fluid model can be found in our technical report [13].
The average downloading speed of peers at time $t$ is

$$
u(t)=\mu \frac{\eta x(t)+y(t)}{x(t)}=\mu\left(\eta+\frac{y(t)}{x(t)}\right) .
$$

We use the tracker trace to validate the torrent evolution model. Similar to the peer arrival rate, the modeling results fit the trace better for torrents with larger populations. Figure 7(a) shows the torrent evolution by both our fluid model and the analysis results of a typical torrent in the trace. The figure shows that the number of downloaders increases exponentially in a short period of time after the torrent's birth (the flash crowd period), and then decreases exponentially, but at a slower rate. The number of seeds also increases exponentially at first, and then decreases exponentially at a slower rate. The peak time of the number of seeds lags behind that of the number of downloaders. As a result, $u(t)$ increases until the torrent is dead, and the resources of seeds cannot increase in proportion to service demand. Furthermore, due to the random arrival of downloaders and the random departure of seeds, average downloading performance fluctuates significantly when the number of peers in the torrent is small, as shown in Figure 7(b).

Figure 8(a) shows the performance variations of the torrent under two kinds of granularities. The instant speed represents the mean downloading speed of all peers in the torrent at that time instant, sampled every half an hour. The average speed represents the average value of the instant speed over the typical downloading time (the average downloading time of all peers). The figure shows that the client downloading speed at different time stages is highly diverse and can affect client downloading time significantly. The reason is that seeds play an important role in the client downloading performance. However, the generation of seeds is the same as the completeness of peer downloading, so the random fluctuation of downloading speed cannot be smoothed in 


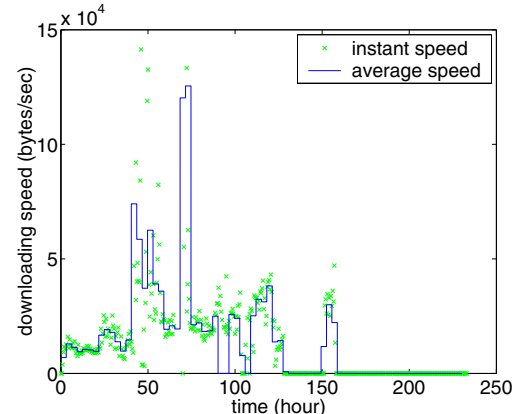

(a) The downloading speed in the lifetime of a typical torrent

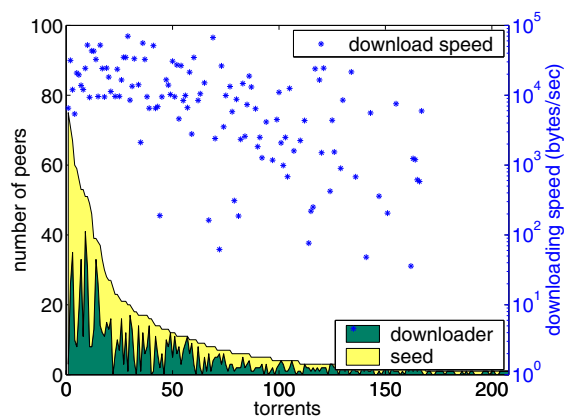

(b) The downloading speed (in log scale) and the number of downloaders/seeds for each torrent at a time instant

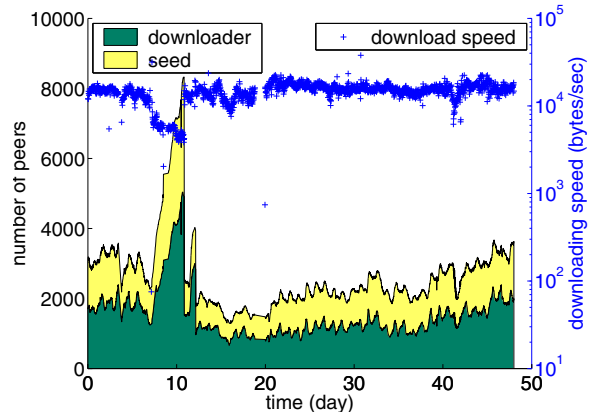

(c) The average downloading speed (in log scale) and the total number of downloaders/seeds for all torrents in the system

Figure 8: Performance variations in BitTorrent systems

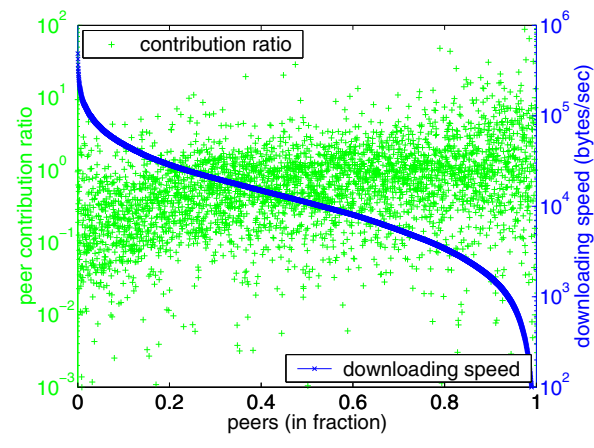

(a) The peer downloading speed and contribution ratio

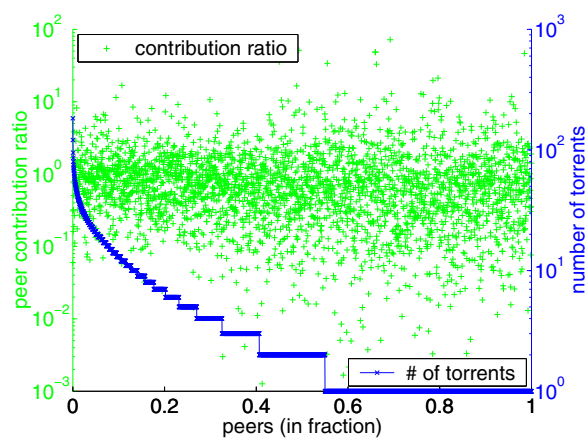

(b) The the number of torrents that each peer involves and the corresponding contribution ratio

Figure 9: Fairness of seed service policy in BitTorrent systems ( $y$-axis is in log scale)

the scale of typical downloading time when the number of peers is small.

Figure 8(b) shows the number of downloaders and seeds (a stack figure), and the average downloading speed for each torrent in the trace at 12:00:01 on 200311-15. In this figure, each point in $x$-axis denotes a torrent, while the left $y$-axis denotes the number of downloaders and seeds in this torrent (stacked), and the right $y$-axis denotes average downloading speed of this torrent. The torrents in the $x$-axis are sorted in nonascending order of the number of downloaders and seeds of torrents. The results at other time instants are similar. In general, peers in torrents with larger population have relatively higher and more stable downloading speed, while the downloading speed in torrents with small populations disperses significantly. When the number of peers in the torrent is small, the client downloading performance is easily affected by the individual behavior of seeds.

Figure 8(c) shows the total number of peers in all torrents (a stack figure) and the average downloading speed of all downloaders in the trace at different time stages.
The average downloading speed of all torrents is shown to be much more stable than that of one torrent. The reason is that the downloader/seed ratio is much more stable due to the large population of the system. This motivates us to balance the service load among different torrents, so that each torrent can provide relatively stable downloading performance to clients in its lifespan.

\subsection{Service Fairness Study in BitTorrent}

In a BitTorrent system, the service policy of seeds favors peers with high downloading speed, in order to improve the seed production rate in the system, i.e., to have these high speed downloaders complete downloading as soon as possible and wish they will then serve other downloaders. In this subsection, we investigate whether this wish comes true in practice.

We define the contribution ratio of a peer as the total uploaded bytes over the total downloaded bytes of the peer. Figure 9(a) shows the peer downloading speed and the corresponding contribution ratio extracted from the trace. In this figure, each point in the $x$-axis denotes a 


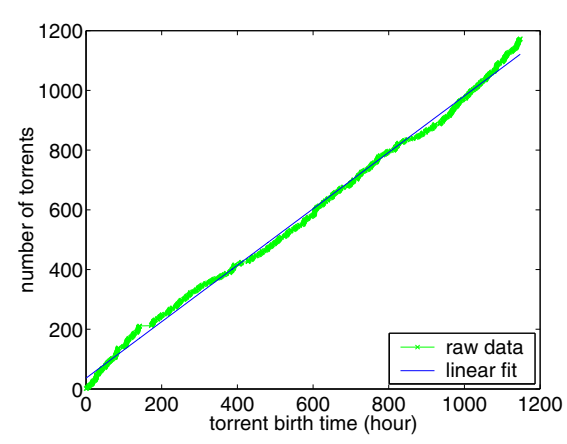

(a) Torrent birth

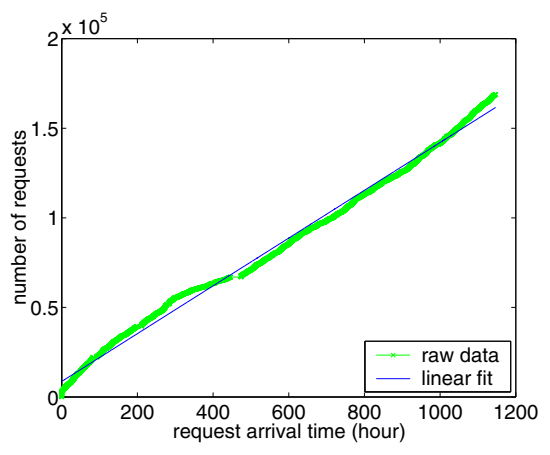

(b) Request arrival of all peers over all torrents

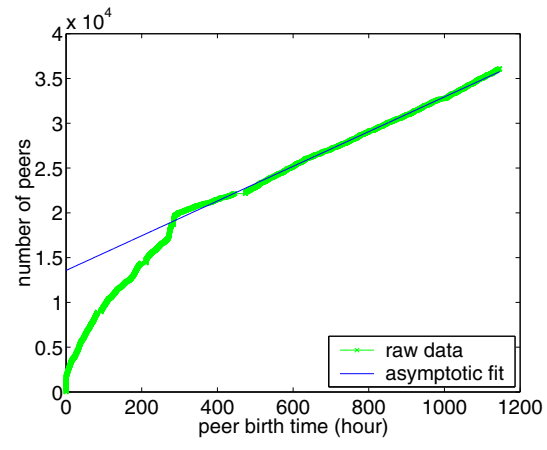

(c) Peer birth

Figure 10: The CDFs of torrent birth, peer request arrival, and peer birth over the trace collection time

peer, while the left $y$-axis denotes the contribution ratio of this peer, and the right $y$-axis denotes the average downloading speed of this peer. On the $x$-axis, peers are sorted in non-ascending order of their contribution ratios. The figure shows the rough trend that the peer contribution ratio increases when the downloading speed decreases. That is, the higher the downloading performance peers have, the less uploading service they actually contribute. This indicates that peers with high speed finish downloading quickly and then quit the system soon, which defeats the design purpose of the seed service policy.

Figure 9(b) shows the number of torrents that each peer involves and its corresponding contribution ratio (plotted in the similar way as that of Figure 9(a)). The figure shows no distinguishable correlation between the two, indicating that the main reason for seeds to leave old torrents is not to start new downloading tasks.

In summary, we observe that the BitTorrent's biased seed service policy in favor of high speed downloaders really affects the fairness to peers in downloading, and an incentive mechanism is needed to encourage seeds to contribute.

\section{Modeling Multiple Torrents in BitTor- rent Systems}

In the previous section, we have shown that client performance fluctuates significantly in single-torrent systems, but is very stable when aggregated over multiple torrents. Based on this observation, in this section, we study the correlation among multiple torrents through modeling and trace analysis, aiming to look for solutions to enable inter-torrent collaboration.

Although different torrents are independent from each other in the current BitTorrent systems, they are inherently related by peers that request multiple torrent files. A peer may download a torrent file, serve as a seed for that torrent for a while, and then go offline to sleep for some period of time. The peer may return sometime later and repeat the activities above. Thus, a peer's lifecyle consists of a sequence of downloading, seeding, and sleeping activities. If a peer stops using BitTorrent for a long time that is much longer than its typical sleeping time, we consider the peer as dead.

In the current BitTorrent systems, a peer is encouraged to exchange file chunks with other peers that are downloading the same file instead of serving old torrent files it has downloaded. Thus, in our model, we assume each peer joins (downloading and seeding) each torrent at most once, and joins one torrent at a time. Having these assumptions, we start to characterize peers in multiple torrents.

\subsection{Characterizing the Peer Request Pat- tern}

In the multi-torrent environment, both torrents and peers are born and die continuously. Figure 10(a) shows the $\mathrm{CDF}$ of torrent birth in the trace (indicated by raw data) and our linear fit. The average torrent birth rate (denoted as $\lambda_{t}$ in the following context) is about 0.9454 torrent per hour. Figure 10(b) shows the CDF of torrent request arrivals (for all peers over all torrents) and our linear fit. We define the torrent request rate as the number of downloading requests for all torrents per unit time in the multi-torrent system, denoted as $\lambda_{q}$ in the following context. Although the peer arrival rate of a single-torrent system decreases exponentially as shown in Figure 1, the torrent request rate in the multi-torrent system is almost a constant, about 133.39 per hour.

Since both the torrent birth rate and torrent request rate are almost constant, it is natural to assume that the peer birth rate (denoted as $\lambda_{p}$ in the following context) is also a constant. A peer is born when it appears in the system for the first time. However, as shown in Figure 10(c), 


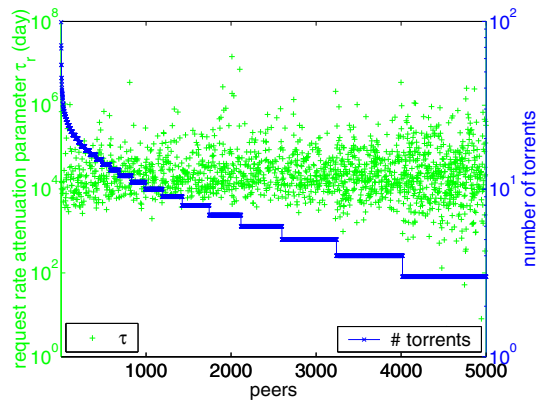

(a) The attenuation of peers' requesting rates and number of torrents peers request ( $y$-axis is in log scale)

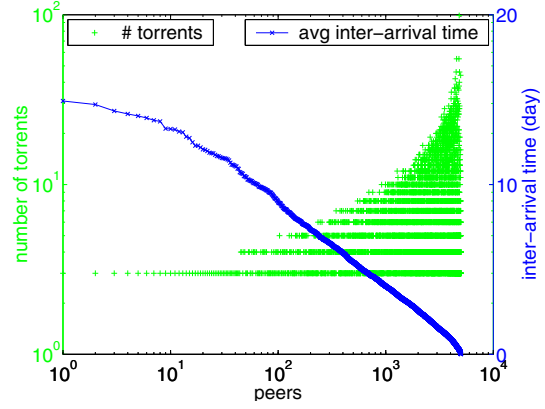

(b) The inter-arrival time of peers' requests and number of torrents they join (in log-log scale)

Figure 11: The request pattern of peers

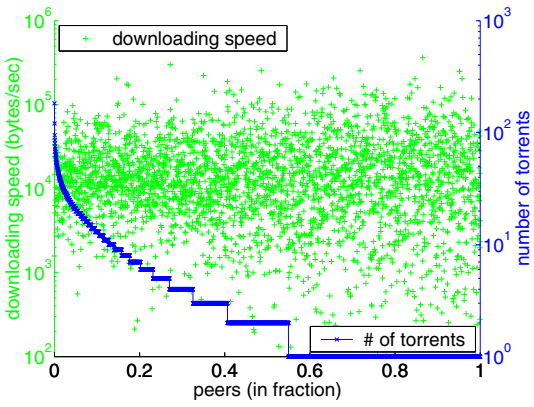

(c) The downloading speed and number of torrents peers join ( $y$-axis is in log scale) the peer birth rate is high at the beginning of the trace collection duration, and then converges to a constant rate asymptotically. The reason is that peers appear in the trace for the first time may actually be born before the trace collection, and the number of such peers decreases quickly after the trace collection starts. Thus, we take the asymptotic birth rate as the real birth rate of peers, which is about 19.37 per hour.

The constant peer birth rate and torrent request rate indicate that each peer only joins a limited number of torrents. However, the request rate of a peer might still change over time. We define the peer request rate as the number of requests a peer submits for different torrents per unit time. Assume the peer request rate can be expressed as

$$
r(t)=r_{0} e^{-\frac{t}{\tau_{r}}}
$$

where $t$ is the time duration after the peer is born, $r_{0}$ is the initial request rate, and $\tau_{r}$ is the attenuation parameter of the request rate. When $\tau_{r} \rightarrow \infty$, the peer has a constant request rate; when $\tau_{r}<0$, the peer has an increasing request rate.

The inter-arrival time between two successive requests of a peer $\delta t$ is $\frac{1}{r(t)}$. Thus, we have

$$
\log \delta t=-\log r_{0}+\frac{t}{\tau_{r}}
$$

We extract $\delta t$ and $t$ from the trace for each peer requesting multiple torrents, and use linear regression to compute $\log r_{0}$ and $\frac{1}{\tau_{r}}$. Figure 11(a) shows the number of torrents that each peer requests and the corresponding $\tau_{r}$. In this figure, each point in the $x$-axis denotes a peer, while the left $y$-axis denotes the $\tau_{r}$ value of this peer, and the right $y$-axis denotes the number of torrents this peer participates. In $x$-axis, peers are sorted in non-ascending order of the number of torrents they join. As shown in the figure, the value of parameter $\tau_{r}$ in Equation 4.10 is very large, with the mean value of about 77 years, which implies that the average request rates of peers do not change significantly over time. Further, $\tau_{r}$ is independent of the number of torrents that peers join. Thus, we can assume that the request processes of peers are Poisson-like processes with constant average request rates.

Figure 11(b) shows the average inter-arrival time of torrent requests for peers requesting multiple torrent files (plotted in the similar manner as that of Figure 11(a)). As shown in the figure, it is intuitive to find that the upper bound of the number of torrents each peer requests increases with the decrease of inter-arrival time. However, for peers with similar request rates, the number of torrents they request are very diverse, since they stay in the system for different time durations. Figure 11(c) further plots the downloading speed versus the number of torrents peers join (plotted in the similar manner as that of Figure 11(a)). There is no strong correlation between the two for peers with downloading speed $>1 \mathrm{~KB}$ per second. This implies that for peers whose downloading speed is large enough, the numbers of torrent files different peers request do not depend on their request rates and their downloading speed.

Thus, we assume that a peer joins a new torrent with probability $p$. For $N$ peers in the system, during their whole lifecycles, there are $N p^{m-1}$ peers that request at least $m$ torrents. Ranking peers in non-ascending order of the number of torrents they join, the number of torrents that a peer ranked $i$ joins is

$$
m=1+\frac{\log i-\log N}{\log p}
$$

In addition, a peer has the probability $1-p$ to download exactly 1 file, probability $p(1-p)$ to download exactly 2 files, and probability $p^{k-1}(1-p)$ to download exactly $k$ files. So the mean number of torrents that a 


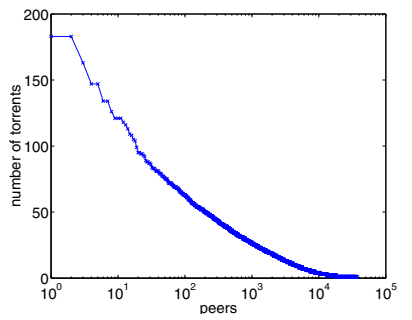

(a) For all peers in the trace

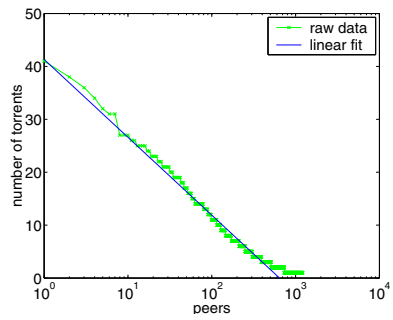

(b) For peers born in the middle of trace collection time

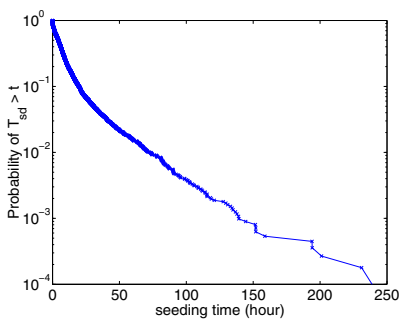

(a) The probability distribution of seeding time

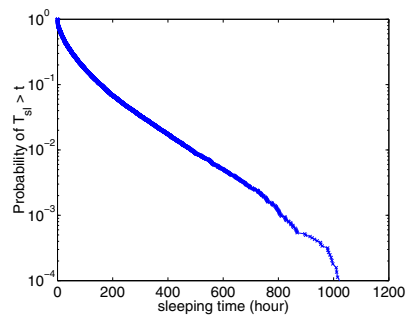

(b) The probability distribution of sleeping time
Figure 12: Torrent involvement of peers ( $x$-axis is in $\log$ scale)

peer joins is:

$$
\bar{m}=\sum_{k=1}^{\infty} k p^{k-1}(1-p)=\frac{1}{1-p} .
$$

Figure 12(a) shows the distribution of number of torrent files that each peer downloads in the trace. The curve in the figure is a little convex, deviating from what Equation 4.12 predicts (a straight line when $x$-axis is in log scale). The reason is that the number of torrents joined by peers born before the trace collection is under-estimated, since some of these requests cannot be recorded in the trace. A similar situation exists for peers that are not dead before the end of trace collection.

Figure 12(b) shows the distribution of number of torrents joined by each peer that was born in the middle of the trace collection duration (indicated by raw data) and our linear fit. The curve fits Equation 4.12 very well, and we estimate from the analysis that $p \approx 0.8551$, while the average number of torrents each peer joins is about 7.514 .

To verify the probability model we use in the above analysis, we estimate $p$ in another way as follows. Assuming the peer birth rate is $\lambda_{p}$ and the torrent request rate is $\lambda_{q}$, since each peer joins $\frac{1}{1-p}$ torrents during its lifetime in average, we have

$$
\lambda_{q}=\frac{1}{1-p} \lambda_{p}
$$

Based on the peer request arrival rate and the peer birth rate we derived before (see Figure 10(b) and 10(c)), we have $p=1-\frac{\lambda_{p}}{\lambda_{q}}=0.8548$. This is very close to the value we got from Equation 4.12, 0.8551, meaning that there are more than $85 \%$ peers joining multiple torrents.

Having characterized the torrent request pattern of peers, finally we consider the distribution of the seeding time and the sleeping time of peers. According to our fluid model, $\frac{1}{\gamma}$ represents the average seeding time. Figure 13(a) and 13(b) show the probability distribution functions of the peer seeding time and the peer sleeping
Figure 13: The seeding time and sleeping time of peers ( $y$-axis is in log scale)

time in the system. Note that the $y$-axis is in log scale. Both the peer seeding time and sleeping time roughly follow the exponential distribution with probability density function $f_{s d}(t)=\frac{1}{\tau_{s d}} e^{-\frac{t}{\tau_{s d}}}$, and $f_{s l}(t)=\frac{1}{\tau_{s l}} e^{-\frac{t}{\tau_{s l}}}$, respectively. Based on the trace analysis, we estimate $\tau_{s d}=\frac{1}{\gamma}=8.42$ hours, and $\tau_{s l}=58.32$ hours.

\subsection{Characterizing the Inter-Torrent Rela- tion}

In this part we study how different torrents are connected through peers that download multiple files, based on our previously verified assumptions.

For simplification, we consider a homogeneous multitorrent environment where all torrents and peers have the same $\lambda_{0}, \tau, \mu, c, \eta, \gamma$, and average sleeping time. We denote each torrent in the system as torrent $i$ with birth time $t_{i}(1 \leq i<\infty)$. For any two torrents that are born successively, torrent $j$ first born and torrent $i$ born next, we have $i=j+1$ and $t_{i}>t_{j}$.

Assume the probability that a peer selects torrent $i$ at time $t_{0}$ as its $k$-th torrent is $P_{i}^{k}\left(t_{0}\right), P_{i}^{k}\left(t_{0}\right)=0$ when $t_{i}>t_{0}$. We also denote $P_{i}^{1}\left(t_{0}\right)$ as $P_{i}\left(t_{0}\right)$ for simplicity. Without loss of generality, we assume that the most recently born torrent by time $t_{0}$ is torrent 1 , and $P_{i}\left(t_{0}\right)$ satisfies

$$
P_{i}\left(t_{0}\right)=\frac{e^{-\frac{t_{0}-t_{i}}{\tau}}}{\sum_{j=1}^{\infty} e^{-\frac{t_{0}-t_{j}}{\tau}}},
$$

where $t_{j}=t_{0}-\frac{j}{\lambda_{t}}, 1 \leq j<\infty$. Thus, we have

$$
\begin{aligned}
P_{i}\left(t_{0}\right)=\frac{e^{-\frac{i}{\lambda_{t} \tau}}}{\sum_{j=1}^{\infty} e^{-\frac{j}{\lambda_{t} \tau}}} & =\left(e^{\frac{1}{\lambda_{t} \tau}}-1\right) e^{-\frac{i}{\lambda_{t} \tau}} \\
& =\left(e^{\frac{1}{\lambda_{t} \tau}}-1\right) e^{-\frac{t_{0}-t_{i}}{\tau}}
\end{aligned}
$$

For a peer that requests its $k$-th torrent file, the peer does not select the torrents that it has requested. Assuming

$$
P_{i}^{k}\left(t_{0}\right)=\alpha_{k} P_{i}\left(t_{0}\right)
$$




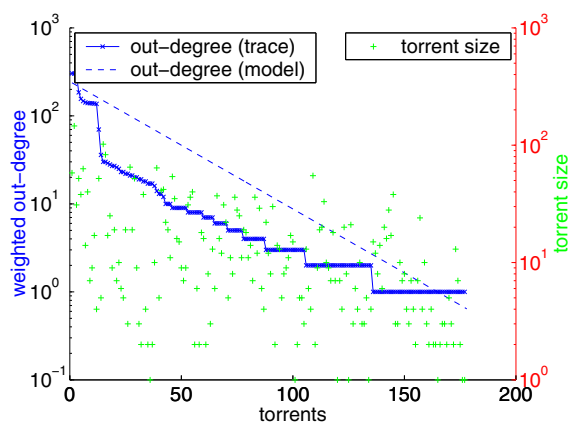

(a) Weighted out-degree

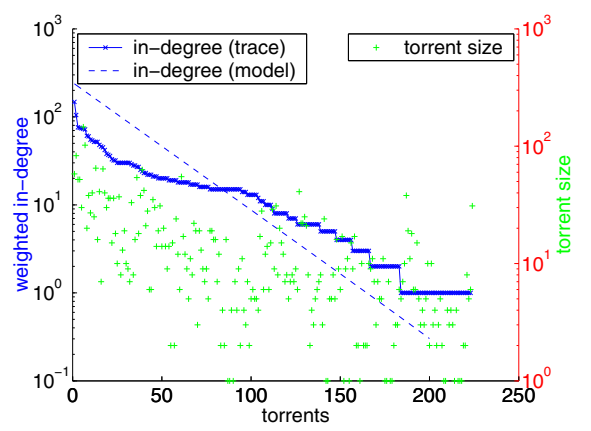

(b) Weighted in-degree

Figure 14: The inter-torrent relation ( $y$-axis is in log scale)

the peer arrival rate of a torrent can be expressed as

$$
\begin{aligned}
\lambda(t) & =\alpha \lambda_{q} P_{i}\left(t_{0}\right) \\
& =\frac{\alpha}{1-p} \lambda_{p}\left(e^{\frac{1}{\lambda_{t} \tau}}-1\right) e^{-\frac{t-t_{i}}{\tau}}
\end{aligned}
$$

where $\alpha=\sum_{k=1}^{\infty} \alpha_{k} p^{k-1}(1-p)$. When $\lambda_{t} \gg r$, we have $\alpha_{k} \approx 1$ and $\alpha \approx 1$. Comparing Equation 3.1 with 4.18, we have $\lambda_{0}=\frac{\alpha}{1-p} \lambda_{p}\left(e^{\frac{1}{\lambda_{t} \tau}}-1\right)$.

Considering that a peer in a torrent may have downloaded files from other torrents, we can model the relationship among different torrents in the P2P system as a directed graph. Each node in the graph represents a torrent. A directed edge from torrent $i$ to torrent $j$ denotes that some peers in torrent $i$ have downloaded the file from torrent $j$, and thus have the potential to provide service to peers in torrent $j$, even though they are not in torrent $j$ currently. The weight of the directed edge $W_{i, j}$ represents the number of such peers. For simplicity, we define $W_{i, i}=0$.

The graph changes dynamically over time. Now let us consider the graph at time $t_{0}$. During time $[t, t+d t]$, $t_{j} \leq t<t_{0}$, there are $\lambda(t) d t$ peers who joined torrent $j$. Let $k(t)=\left\lfloor r\left(t_{0}-t\right)\right\rfloor$. During time $\left[t, t_{0}\right]$, these peers can download up to $k(t)-1$ torrents completely in addition to torrent $j$ and may request (or be requesting) the next torrent at time $t_{0}$. If torrent $i$ is not requested before the last requests during time $\left[t, t_{0}\right]$, the probability of such events is

$$
Q_{i}(t)=p \times \prod_{l=1}^{k(t)-1} p \times\left(1-\alpha_{l} P_{i}\left(t+\frac{l}{r}\right)\right) .
$$

When $i \neq j$, we have

$$
W_{i, j}=\int_{t_{j}}^{t_{0}} \lambda(t) d t \times Q_{i}(t) \times \alpha_{k(t)} P_{i}\left(t+\frac{k(t)}{r}\right) .
$$

Therefore, the weighted out-degree of torrent $i$ represents the total potential capability its peers can provide to peers in other torrents, denoted as $S P_{i}$, where

$$
S P_{i}=\sum_{j=1}^{\infty} W_{i, j}
$$

Correspondingly, the weighted in-degree of torrent $i$ represents the total potentials its peers can get from peers in other torrents, denoted as $S G_{j}$, where

$$
S G_{j}=\sum_{i=1}^{\infty} W_{i, j}
$$

Figure 14(a) and 14(b) show the weighted out-degree and weighted in-degree at a time instant based on trace analysis and our probability model, respectively. In the figures, each point in the $x$-axis denotes a torrent, sorted in non-ascending order of weighted out-degree or weighted in-degree. The right $y$-axis in the figures denotes torrent size, the number of peers in the torrent at this time instant. In general, torrents with more peers tend to have large out-degree and in-degree. The weighted out-degree and in-degree distribution according to our trace analysis follows power law rules roughly. It deviates from our model somewhat because of the heterogeneity of torrents in the real system.

In the multi-torrent environment, old peers that had downloaded the file from a torrent may come back for other torrent files, and the lifespan of this torrent can be extended if these old peers are willing to provide service. Assume the request arrival rate of this torrent is $\lambda(t)$ and $\lambda(t)=0$ when $t<0$. If we consider both new requesting peers and old returning peers, the peer arrival rate of the torrent is

$$
\begin{aligned}
\lambda^{\prime}(t) & =\sum_{l=0}^{k(t)} p^{l} \lambda\left(t-\frac{l}{r}\right)=\sum_{l=0}^{k(t)} p^{l} \lambda_{0} e^{-\frac{t-\frac{l}{r}}{\tau}} \\
& =\lambda_{0} e^{-\frac{t}{\tau}} \frac{q^{k(t)+1}-1}{q-1}
\end{aligned}
$$

where $k(t)=\lfloor r t\rfloor$ and $q=p e^{\frac{1}{r \tau}}(q>1$ based on our trace analysis). 
When $\lambda^{\prime}(t)<\gamma$, the torrent is truly dead. The lifespan of a torrent without inter-torrent collaboration is $T_{\text {life }}=$ $\tau \log \left(\frac{\lambda_{0}}{\gamma}\right)$. Denoting the lifespan of the torrent with intertorrent collaboration as $T_{\text {life }}^{\prime}$, then $\lambda^{\prime}\left(T_{\text {life }}^{\prime}\right)=\gamma$, we have

$$
\begin{aligned}
\log \gamma & =\log \lambda_{0}-\frac{T_{l i f e}^{\prime}}{T_{l i f e}^{\prime}}+\log \left(q^{k\left(T_{\text {life }}^{\prime}\right)+1}-1\right)-\log (q-1) \\
& \approx \log \lambda_{0}-\frac{\left.\left.T_{l i f e}^{\prime}\right)+1\right) \log q-\log (q-1)}{T_{l i f e}^{\prime}}+k\left(T_{\text {life }}^{\prime}\right) \log q+\log \frac{q}{q-1} .
\end{aligned}
$$

It leads to $\log \left(\frac{\lambda_{0}}{\gamma} \frac{q}{q-1}\right) \approx\left(\frac{1}{\tau}-r \log q\right) T_{\text {life }}^{\prime}$. Thus

$$
\begin{aligned}
T_{\text {life }}^{\prime} & \approx \frac{\tau \log \left(\frac{\lambda_{0}}{\gamma} \frac{q}{q-1}\right)}{1-\tau r \log q}=\frac{\tau \log \left(\frac{\lambda_{0}}{\gamma} \frac{q}{q-1}\right)}{\tau r \log \frac{1}{p}} \\
& >\frac{T_{\text {life }}}{\tau r \log \frac{1}{p}}=\beta T_{\text {life }} .
\end{aligned}
$$

According to the trace analysis and our modeling, $\beta=$ $\frac{1}{\tau r \log \frac{1}{p}} \approx 6$. So we have

$$
R_{\text {fail }}^{\prime}=e^{-\frac{T_{l i f e}^{\prime}}{\tau}}<R_{f a i l}^{\beta} \approx R_{f a i l}^{6} .
$$

Comparing Equation 4.25 with 3.6, we can see that inter-torrent collaboration is much more effective than decreasing the seed leaving rate $\gamma$ for reducing downloading failure ratio. Decreasing seeds leaving rate has polynomial effect, while inter-torrent collaboration has exponential effect. For example, if the current downloading failure rate is 0.1 , and seeds can be stimulated to stay 10 times longer (i.e., $\gamma$ will decrease 10 times), then the downloading failure rate will decrease 10 times to 0.01 . However, by inter-torrent collaboration, the downloading failure ratio can be as low as $0.1^{6}=10^{-6}$. The reason is that extending seed staying time only increases the service time for peers that arrive close to the seed generation time. With the passage of time, the peer arrival rate decreases exponentially, and finally the seed serving time will not be long enough for newly arriving peers. On the other hand, by exploiting inter-torrent collaboration, peers that have downloaded the file may return multiple times during a much longer period, and the downloading failure ratio can be significantly reduced to near zero.

\section{A Discussion of Multi-Torrent Collabo- ration Systems}

In this section, we discuss the principle of a system design for multi-torrent collaboration. A more detailed discussion can be found in [13]. The system design and implementation are ongoing.

\subsection{Tracker Site Overlay}

In BitTorrent systems, peers in different torrents cannot collaborate because they cannot find and communicate

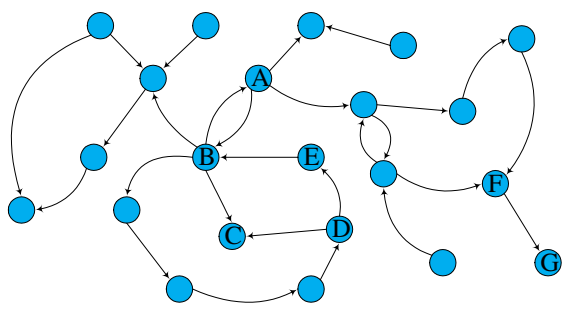

Figure 15: Tracker site overlay

with each other. The inter-torrent relation graph presented in Section 4.2 motivates us to organize the tracker sites of different torrents into an overlay network to help the peers sharing different files find each other and coordinate the collaboration among these peers. In such an overlay network, each tracker site maintains a NeighborOut Table and a Neighbor-In Table to record the relationship with its neighboring torrents. The Neighbor-Out Table records the torrents that its peers can provide service to. The Neighbor-In Table records the torrents whose peers can provide service to this torrent. When a peer $q$ joins a new torrent $A$, it uploads to its tracker site the information about from which torrents it had downloaded files previously. Then $A$ 's tracker site forwards this information to the tracker sites of those torrents where $q$ had downloaded files from. By doing so, the torrents that are created independently by different content providers are connected together to form a tracker site overlay, as shown in Figure 15. Tracker site overlay also provides a built-in mechanism to search content among multiple torrents. Currently, BitTorrent users have to rely on Webbased search engines to look for the content they want to download.

\subsection{Exchange Based Incentive for Multi- torrent Collaboration}

BitTorrent assumes each peer is selfish, and exchanges file chunks with those peers that provide it the best service. The incentive mechanism in BitTorrent systems is instant, because each peer must get corresponding benefit at once for the service it provides. For multi-torrent collaboration, an exchange based mechanism can be applied for instant collaboration through the tracker site overlay, which still follows the "tit-for-tat" idea.

First, peers in adjacent torrents in the overlay can exchange file chunks directly, such as torrent $A, B$ in Figure 15. Second, if there exists a cycle among several torrents, then peers in adjacent torrents can exchange file chunks through the coordination of the tracker site overlay, such as torrent $B, C, D, E$ in Figure 15. More specifically, when a peer $q$ wants to get service from 
peers in other torrents, it sends a request to its tracker site with its list of downloaded files. Then the tracker forwards its request to the trackers in its Neighbor-In Table. These tracker sites then search their tables to find qualified peers, with whom this peer can exchange file chunks to get service.

When a peer $q$ wants to get service from peers in other torrents and it has no service to exchange, it may join these torrents temporarily and download some chunks of the files, even if it does not want these files itself. Through the coordination of corresponding tracker sites, the peer can provide uploading service for these chunks only, and attribute its service contribution to the peers it wants to get service from, so that these peers can get benefit from the peers that $q$ serves and offer $q$ the service it needs. Since a file chunk can be served to multiple peers in the system, this method is very effective and the overhead is trivial. Research [6,9] presents similar idea of using file exchange as an incentive for P2P content sharing. Different from these studies, our system aims to share bandwidth as well as content across multiple P2P systems.

\section{Conclusion}

BitTorrent-like systems have become increasingly popular for object distribution and file sharing, and have contributed to a large amount of traffic on the Internet. In this paper, we have performed extensive trace analysis and modeling to study the behaviors of such systems. We found that the existing BitTorrent system provides poor service availability, fluctuating downloading performance, and unfair services to peers. Our model has revealed that these problems are due to the exponentially decreasing peer arrival rate and provides strong motivation for inter-torrent collaborations instead of simply giving seeds incentives to stay longer. We also discuss the design of a new system where the tracker sites of different torrents are organized into an overlay to facilitate inter-torrent collaboration with the help of an exchange based incentive mechanism.

\section{Acknowledgments}

We would like to thank our shepherd, Keith W. Ross, and the anonymous reviewers for their constructive comments. We are grateful to Oliver Spatscheck for many insightful discussions on this topic. Mikel Lzal helped us get access to their BitTorrent traces and William L. Bynum provided valuable comments on an early draft of the paper. This work is partially supported by the National Science Foundation under grants CNS-0098055, CNS-0405909, and CNS-0509054/0509061.

\section{References}

[1] http://www.gnutelliums.com/.

[2] http://www.kazaa.com/.

[3] http://www.edonkey2000.com/.

[4] Hack kazaa participation level - the easy answer. http://www.davesplanet.net/kazaa/.

[5] Adar, E., And B.Huberman. Free riding on gnutella. Tech. rep., Xerox PARC, August 2000.

[6] Anagnostakis, K. G., and Greenwald, M. B. Exchangebased incentive mechanisms for peer-to-peer file sharing. In Proc. of IEEE ICDCS (March 2004).

[7] Bellissimo, A., Levine, B. N., And Shenoy, P. Exploring the use of BitTorrent as the basis for a large trace repository. Tech. Rep. 04-41, University of Massachusetts Amherst, June 2004.

[8] Cohen, B. Incentives build robustness in BitTorrent. In Proc. of Workshop on Economics of Peer-to-Peer Systems (May 2003).

[9] Cox, L. P., And Noble, B. D. Samsara - honor among thieves in P2P storage. In Proc. of ACM SOSP (October 2003).

[10] Cranor, C., Johnson, T., And Spatscheck, O. Gigascope: a stream database for network applications. In Proc. of ACM SIGMOD (June 2003).

[11] Ge, Z., Figueiredo, D. R., Jaiswal, S., Kurose, J., ANd TOWSLEY, D. Modeling peer-peer file sharing systems. In Proc. of IEEE INFOCOM (March 2003).

[12] Gummadi, K. P., Dunn, R. J., Saroiu, S., Gribble, S. D., LEVY, H. M., AND ZAHORJAN, J. Measurement, modeling, and analysis of a peer-to-peer file-sharing workload. In Proc. of ACM SOSP (October 2003).

[13] Guo, L., Chen, S., Xiao, Z., Tan, E., Ding, X., And ZHANG, $X$. Measurements, analysis, and modeling of BitTorrent systems. Tech. Rep. WM-CS-2005-08, College of William and Mary, July 2005.

[14] IZal, M., Urvoy-Keller, G., Biersack, E., Felber, P., Hamra, A. A., And Garc'Es-ERICE, L. Dissecting BitTorrent: Five months in a torrent's lifetime. In Proc. of the 5 th $\mathrm{An}$ nual Passive \& Active Measurement Workshop (April 2004).

[15] Massoulie, L., And Vojnovic, M. Coupon replication systems. In Proc. of ACM SIGMETRICS (June 2005).

[16] PARKer, A. The true picture of peer-to-peer file sharing. http://www.cachelogic.com, 2004.

[17] Pouwelse, J., Garbacki, P., Epema, D., And Sips, H. The BitTorrent P2P file-sharing system: Measurements and analysis. In Proc. of the 4th International Workshop on Peer-to-Peer Systems (February 2005).

[18] QIU, D., AND SRIKANT, R. Modeling and performance analysis of BitTorrent-like peer-to-peer networks. In Proc. of ACM SIGCOMM (August 2004).

[19] Saroiu, S., Gummadi, K., Dunn, R., Gribble, S., And LEVY, H. An analysis of Internet content delivery systems. In Proc. of USENIX OSDI (December 2002).

[20] Saroiu, S., Gummadi, K., And Gribble, S. A measurement study of peer-to-peer file sharing systems. In Proc. of ACM/SPIE MMCN (January 2002).

[21] Sherwood, R., Braud, R., and Bhattacharjee, B. Slurpie: A cooperative bulk data transfer protocol. In Proc. of IEEE INFOCOM (March 2004).

[22] SRipanidKulchai, K., MAGgs, B., AND Zhang, H. Efficient content location using interest-based locality in peer-to-peer systems. In Proc. of IEEE INFOCOM (March 2003).

[23] Yang, X., AND D. Veciana, G. Service capacity of peer to peer networks. In Proc. of IEEE INFOCOM (March 2004). 